Software Tools for Technology Transfer manuscript No.

(will be inserted by the editor)

\title{
Model Checking Java Programs Using Java PathFinder
}

Klaus Havelund, Thomas Pressburger

NASA Ames Research Center,

Recom Technologies,

Moffett Field, California, USA.

e-mail: $\{$ havelund, $t$ tp $\} \oplus$ ptolemy . arc . nasa.gov

url: http://ase.arc.nasa.gov/ \{havelund, ttp $\}$

March 12, 1999

Abstract. This paper describes a translator called JAVA PathFinder from JaVA to Promela, the "programming language" of the SPIN model checker. The purpose is to establish a framework for verification and debugging of JAVA programs based on model checking. This work should be seen in a broader attempt to make formal methods applicable "in the loop" of programming within NASA's areas such as space, aviation, and robotics. Our main goal is to create automated formal methods such that programmers themselves can apply these in their daily work (in the loop) without the need for specialists to manually reformulate a program into a different notation in order to analyze the program. This work is a continuation of an effort to formally verify, using SPIN, a multi-threaded operating system programmed in LISP for the Deep-Space 1 space craft, and of previous work in applying existing model checkers and theorem provers to real applications.

Key words: Program verification - JAVA - model checking - SPIN - concurrent programming - assertions deadlocks. 


\section{Introduction}

In this paper we describe JaVa PathFinder (JPF), a translator from JAVA to PROMELA, the programming language of the SPIN model checker. The purpose is to establish a framework for verification and debugging of JAVA programs based on model checking. The work should be seen in a broader attempt to make formal methods applicable "in the loop" of programming within NASA's areas such as space, aviation, and robotics. Our main long term goal is to create an automated formal methods workbench for JAVA programming such that programmers themselves can apply them in their daily work (in the loop) without the need for specialists to manually reformulate a program in a different notation in order to analyze it.

The tool we are developing is named after the rover operating on Mars in 1997 called the "Mars PathFinder". Although this mission was generally regarded as a big success, the rover did in fact contain a number of software bugs (causing repeated rebootings and panic at NASA headquarters) that could potentially have been found beforehand using proper verification tools. The JAVA PATHFinder name is a play on words: it finds the paths of a JaVA program that lead to errors.

JAVA $[6,1]$ is a general purpose object-oriented programming language with built in mechanisms for multithreaded programming [16]. It was originally designed to support internet programming, but goes well beyond this domain. JAVA is a relatively simple language compared to $\mathrm{C}++$, and it is regarded as a much safer language, amongst other things due to its automatic garbage collection and lack of general pointers. In spite of its simplicity it appears to be a powerful language.

SPIN [15] is a verification system that supports the design and verification of finite state asynchronous process systems. Programs are formulated in the Promela programming language, which is quite similar to an ordinary programming language, except for certain nondeterministic specification oriented constructs. Processes communicate either via shared variables or via message passing through buffered channels. Properties to be verified are stated as assertions in the code, or as formulae in the linear temporal logic LTL. The SPIN model checker can automatically determine whether a program satisfies a property, and, in case the property does not hold, generate an error trace. SPIN also finds deadlocks.

In an earlier effort we formally verified, using SpIN, a multi-threaded operating system for a spacecraft [9]. The operating system is one component of NASA's New Millennium Remote Agent (RA) [17], an artificial intelligence based spacecraft control system architecture launched October 24, 1998, as part of the Deep-Space 1 mission to an asteroid to validate the potential of artificial intelligence, ion propulsion, and other technologies for future space crafts. The operating system is implemented in a multi-threaded version of COMMON LISP.
The verification effort consisted of hand translating parts of the LisP code into the Promela language of SPIN. A total of 5 errors were identified, a very successful result.

It is often claimed that model checkers of today cannot handle real sized programs, and consequently cannot handle real sized JAVA programs. This is certainly true. However, there are two aspects that make our effort worthwhile anyway. First, by providing an abstraction workbench we will make it possible to cut down the state space of a JAVA program. Second, one can imagine model checking being applied for unit testing, where one focuses on a single class (or a few classes) and puts this (these) in parallel with an aggressive environment represented by a number of threads. Finally, JAVA can be used as a design language, just as Promela.

Few attempts have been made to automatically verify programs written in real programming languages. The most recent attempt we can mention is the one reported in [2], which also translates JAvA programs into Promela, however not handling exceptions or polymorphism as we do. The work in [3] defines a translator from a concurrent extension of a very limited subset of $\mathrm{C}++$. The drawback of this solution is that the concurrency extensions are not broadly used by $\mathrm{C}++$ programmers. In [5] is described what is called Extended static checking, a technique for detecting, at compile-time, common programming errors. The technique uses program verification technology, but feels to a programmer like a type checker. By using an underlying automatic theorem prover, the technique is more semantically thorough than decidable static analysis techniques. At the same time, by only trying to detect certain kinds of errors, not prove the program's correctness, the technique is more automatic than program verification. Finally, [4] describes a theory of translating JAVA to a transition model, making use of static pointer analysis to aid virtual coarsening, which reduces the size of the model.

A significant subset of JAVA version 1.0 is supported by JPF: dynamic creation of objects with data and methods, class inheritance, threads and synchronization primitives for modeling monitors (synchronized statements, and the wait and notify methods), exceptions, thread interrupts, and most of the standard programming language constructs such as assignment statements, conditional statements and loops. However, the translator is still a prototype and misses some features, such as packages, overloading, method overriding, recursion, strings, floating point numbers, static variables and static methods, some thread operations like suspend and resume, and some control constructs, such as the cont inue statement. In addition, arrays are not objects as they are in JAVA, but are modeled using Promela's own arrays to obtain efficient verification. Finally, we do not translate the predefined class library.

Note that many of these features can be avoided by small modifications to the input code. In addition, the tool is currently being improved to cover more of JAVA. 
Despite the omissions, we expect the current version of JpF to be useful on a large class of software. A frontend to the translator checks that the program is in the allowed subset and prints out error messages when not. The translator is developed in COMMON LISP, having a JAVA parser written in MoscowML as front end. Gerard Holzmann supported our efforts by changing the semantics for the Promela unless construct in order to simplify the translation of exceptions.

The paper is organized around an example JAVA program that has been translated automatically by JAVA PATHFinder and verified automatically by SPIN. In Section 2 we describe this program, a bounded buffer. In Section 3 we describe what the resulting Promela code looks like. In Section 4 we present an experiment where we seeded 21 errors into the example program, and ran the SPIN model checker on the code generated by JPF. Section 5 ends with conclusions and suggestions for future work.

\section{The Bounded Buffer Program}

The translation scheme will be illustrated by translation of a complete, small, but non-trivial JAVA program that covers many of the features of JAVA that we can translate. After translation by JPF, SpIN can be applied to prove or disprove that the program satisfies given properties stated as assertions in the program, and that it is deadlock free.

\subsection{The Buffer Class}

The JAVA program that we are interested in verifying properties about is a bounded buffer, represented by a single class. An object of this class can store objects of any kind (objects of subclasses of the general top level class Object). Figure 1 shows the declared interface of this class. It contains a put method, a get method and a halt method. Typically there will be one or more producer threads that call the put method, and one or more consumer threads that call the get method. The halt method can be invoked by a producer to inform consumers that it will no longer produce values to the buffer. Consumers are allowed to empty the buffer safely after a halt, but if a consumer calls the get method after the halt method has been called, and the buffer is empty, an exception object of class HaltException will be thrown. A class is an exception class if it is a subclass of the class Throwable. In particular, class Exception is a subclass of Throwable.

Figure 2 contains the Buffer class annotated with line numbers for later reference. A JAVA class is generally speaking described by a name, a set of data variables, zero or more constructor methods (with different argument types if more than one) with the same name

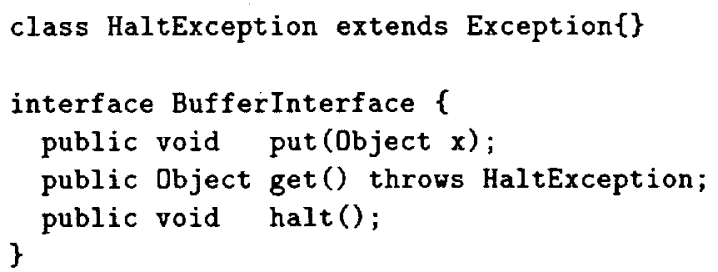

Fig. 1. The JaVA Buffer interface

as the class, and a collection of methods. One of the constructors is executed when an object is created with the new construct. Note that the Buffer class has no such user-defined constructors. The class declares an array of length 3 to hold the objects in the buffer. A cleaner way of writing this program would be to declare a constant "final int SIZE $=3$ " and then refer to SIZE rather than to 3 throughout the program. The translator, however, currently requires an integer literal as the dimension of an array. In addition to the array, a couple of pointers are declared, one pointing to the next free location, and one pointing to the next object to be returned by the get method. The variable usedSlots keeps track of the current number of elements in the buffer. Finally, the variable halted will become true when the halt method is called.

The three methods of the class are all synchronized (note the synchronized keyword). Hence, each of these methods will have exclusive access to the object when executed. That is, when one of these methods is called on the buffer object by a thread, the buffer gets locked to serve that thread, and it is unlocked again at the end of the method call. The put method takes as parameter the object to be stored in the buffer and has no return value (void). It enters a loop testing whether the buffer is full (i.e. having 3 elements) in which case it calls the built in wait method. Calling the wait method within a synchronized method suspends the current thread and allows other threads to execute synchronized methods on the object. Such another thread can then call the not if y method which will wake up an arbitrarily chosen waiting thread to continue past its wait() call. The not ifyAll method wakes up all such waiting threads.

The call of wait is put inside a try construct, which is JAVA's exception handling construct. A general try construct has the form:

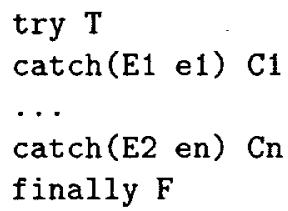

where each $\mathrm{T}, \mathrm{C} 1, \ldots, \mathrm{Cn}, \mathrm{F}$ is a block (a statement or a sequence of statements enclosed by $\{\ldots\}$ ) and each $E i$ is an exception type (class). The body $T$ of the try statement is executed until either an exception is thrown or it finishes successfully. If an exception is thrown, the catch 


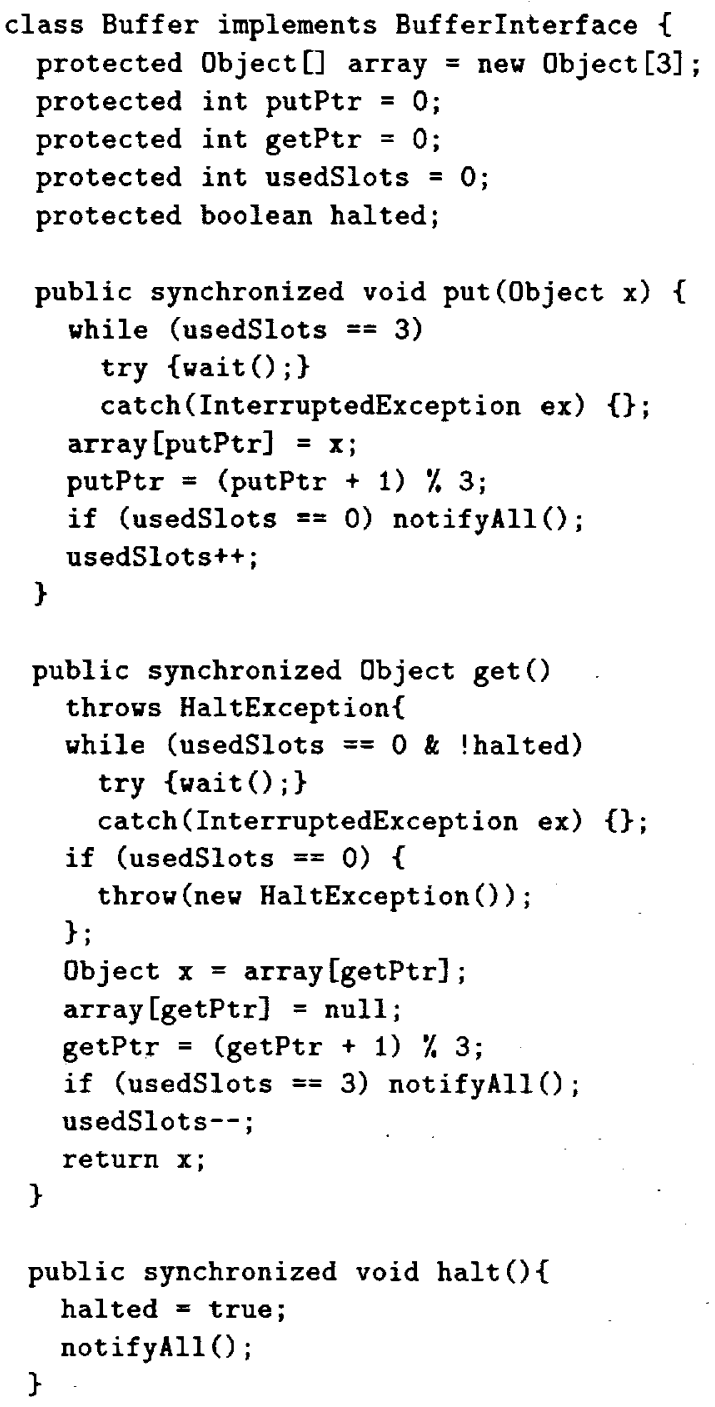

38. $\}$

Fig. 2. The JAVA Buffer class

clauses are examined from top to bottom in order to find one where the thrown exception is of the corresponding class Ei or of a subclass thereof. If such a catch is found, the corresponding block $\mathrm{Ci}$ is executed. If no appropriate catch is found, the exception "flows out" of the try statement into an outer try that might handle it. There can be any number of catch clauses in a try including none. If no catch clause in the method catches the exception, the exception is thrown to the code that invoked this method. If a finally clause is present in a try, its code is executed after all other processing in the try is complete. This happens no matter how the completion was achieved, whether normally, through an exception, or through a control flow statement like return.

Normally an exception is thrown explicitly within a thread using the throw(e) statement, where e is an exception object (a normal object of an exception class which may include data and methods). However, one thread S may throw an exception in another thread $T$ by

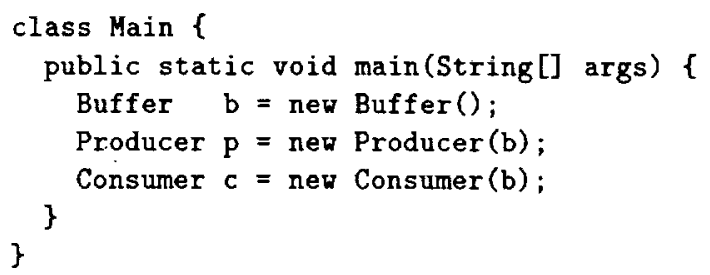

Fig. 3. The JaVA main program

executing $\mathrm{T}$.interrupt (), which throws an

InterruptedException, or T.stop(), which throws a ThreadDeath exception. The try construct around the wait call is supposed to catch exactly such interrupts from other threads. As we sce, nothing is done in this case (lines 11 and 22), but the try statement is necessary in order for the JAVA type checker to accept the program. We shall later see a real use of exceptions. One of the main results in this paper is that we can in fact prove properties of programs that throw exceptions.

When finally the put method gets past the while loop, it is known that the buffer has free space, and the insertion of the new object can be completed. In case the buffer was in fact empty, all waiting consumers are notified.

The get method is a little bit more complicated because it also takes into account whether the buffer has been halted. Basically, it will wait until there is something in the buffer, and return this element, unless the buffer is empty and at the same time has been halted. In this case, a HaltException is thrown. Otherwise, the next buffer element is returned, and producers are notified if the buffer beforehand was full, in which case they may be waiting.

\subsection{Setting up an Environment}

In order to verify properties about this class, without looking at a complete application within which it occurs, we can create a small application using the buffer. We say that we set up an environment consisting of a number of threads accessing the buffer, and then we prove properties about this small system. This can be regarded as unit testing the buffer. Concretely, we shall define two thread classes: a Producer and a Consumer class, and then start the whole system as shown in the main method in Figure 3.

First, in order to illustrate the translator's capabilities to translate inheritance, we define the objects that are to be stored in the buffer, see Figure 4 . A class Attribute is defined, which contains one integer variable. The constructor method with the same name as the class takes a parameter and stores it in this variable. The class AttrData extends this class with another field, and defines a constructor, which takes two parameters, and then calls the super class constructor with the first parameter. 


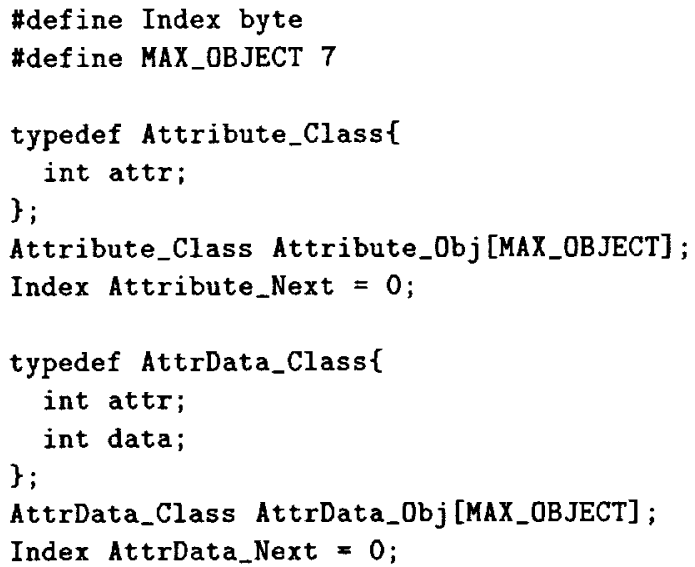

Fig. 7. Object arrays for the Attribute and AttrData classes

as the second statement of the consumer run method. The major advantage of this approach is that we do not need to change the JAVA language, or parse special "specification comments". JAVA itself is used as the specification language. Note, however, that at this point, only the assert method is supported.

\section{Translation}

\subsection{Classes, Inheritance and Object Creation}

The general principle behind the translation is the following. A JAVA class basically consists of data variables and methods. For each new creation of an object, a new set of data variables, a data area, is allocated, and the methods of that object will then work on this newly allocated area. Hence, at any point in time a set of data areas will have been allocated, one for each object not garbage collected. We shall model the set of data areas of a class by an array of records (typedef's in SPIN terminology), one record for each data area. An index variable will point to the next free record in the array, initially having the value 0 (first record in the array). Method definitions are mapped into macro definitions, and method calls are mapped into applications of macros. Note that the translated code shown is the unedited Promela code generated by the translator (except for a couple of omissions).

For illustration, we start with the translation of the simplest classes, namely Attribute and AttrData, which have no methods, but which show inheritance. Figure 7 shows the data areas for the two classes. For each class $\mathrm{C}$ an array named $\mathrm{C}_{-} \mathrm{Obj}$ is defined of size MAX_OBJECT and of type $C_{-} C l a s s$. In addition, a pointer $C_{-}$Index to the next free data area (object) is declared. Because the AttrData class inherits from the Attribute class, it includes the attr variable in addition to the data variable.

Note that this translation puts a limit (MAX_OBJECT) on the number of objects that can be created of a class.

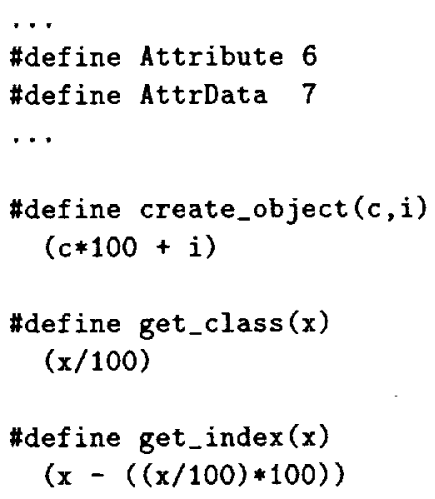

Fig. 8. General object reference operations

A particular object is now referenced by an object reference containing information about which class it concerns (and thereby which array) and which instance (index in the array) it concerns. Because Promela's record concept (typedefs) is not flexible enough for our needs (e.g., field access cannot be applied to a conditional expression) we have decided to represent an object reference $(c, i)$ as the integer $c * 100+i$. The operations (macros) for dealing with these calculations are shown in Figure 8. Each class is given a unique identification which is a number, for example class Attribute is given number 6 .

In order to access the variables in these arrays, macros are defined as shown in Figure 9. These macros are prefixed with the name of the class that defines the variable, which is statically decidable in JAVA at the point where the variable is accessed (in contrast to method calls). However, because JAVA supports polymorphism where an object may belong to a subclass of the statically declared class (for example if it is a parameter to a method) we need to define these variable accessors such that they access the right array. For example, the macro Attribute get_attr for reading the attr variable defined in the Attribute class is defined as a conditional expression over the subclasses.

Both classes have constructors which will be executed when creating new objects. For example, the Java expression "new AttrData $(2,4)$ " will cause a call of the constructor, which in turn will call the constructor of the super class: "Attribute (2)". Constructors are modeled as macros as shown in Figure 10 (slightly simplified for presentation purposes; the real translation also takes into account variable initializations occurring together with variable declarations). The parameter obj will be an object reference denoting the object being constructed.

The construction of a new object such as for example the JAVA statement " $x=$ new AttrData $(2,4)$ " is mapped into the sequence of Promela statements shown in Figure 11 (slightly simplified here with respect to the treatment of the variable $x$ ): one can see how the $x$ is bound to a reference, the next-pointer is incre- 


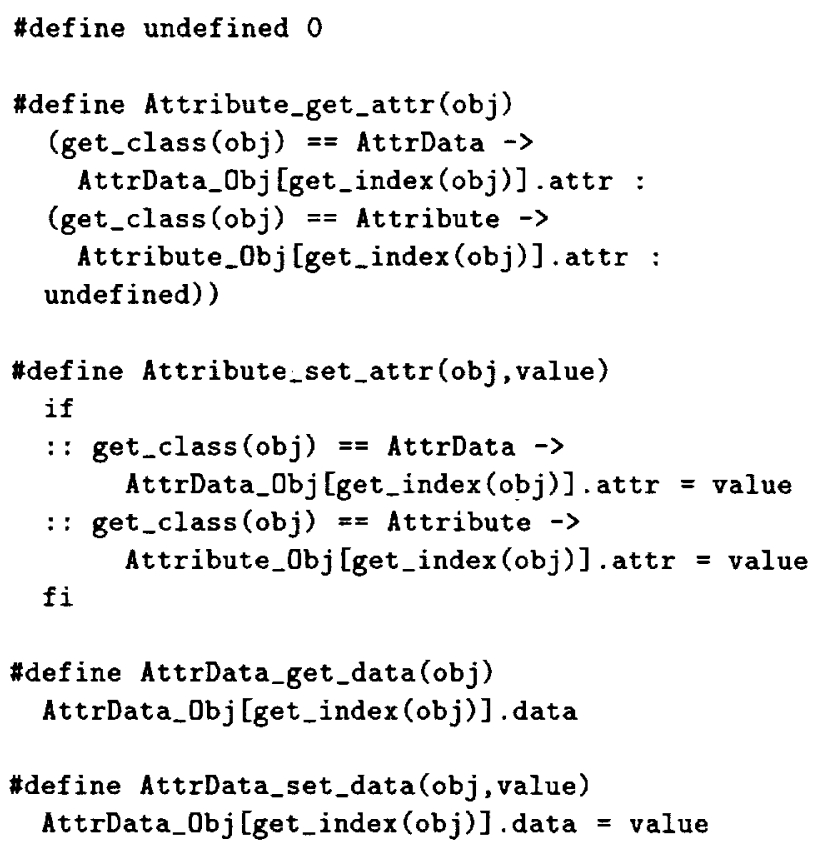

Fig. 9. Variable accessors for the Attribute and AttrData classes

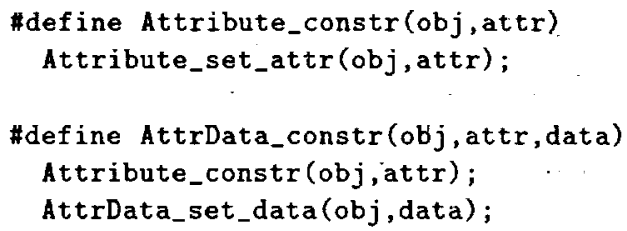

Fig. 10. Constructors for the Attribute and AttrData classes

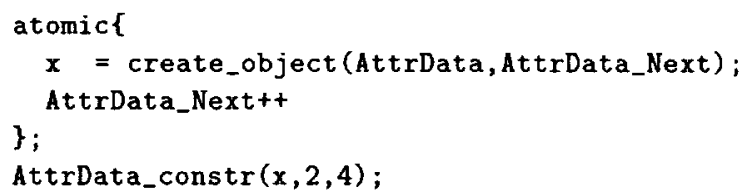

Fig. 11. Translation of $x=$ new AttrData $(2,4)$

mented, and the constructor is applied to the reference now stored in $\mathrm{x}$.

\subsection{Synchronization on Objects}

The Buffer class is more complicated because it contains methods, and because these in addition are synchronized, which means that they must have exclusive access to the object when executing. That is, only one thread at a time may execute any synchronized method on the same object. Therefore, the translation must provide a locking mechanism with which one can lock an object to serve a particular thread that calls a synchronized method on the object. For that purpose some extra variables are inserted into the data area of each object, as shown in Figure 12. In addition to the variables declared in the class (note that JAVA arrays are modeled directly as Promela arrays), it contains the three vari-

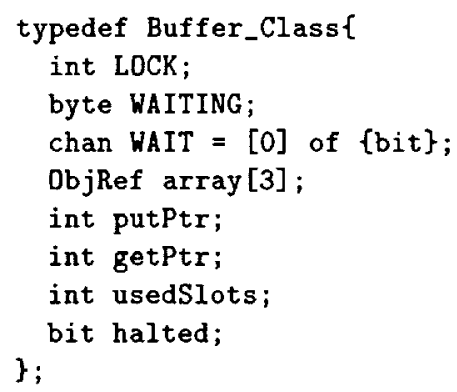

Fig. 12. Object type for the Buffer class

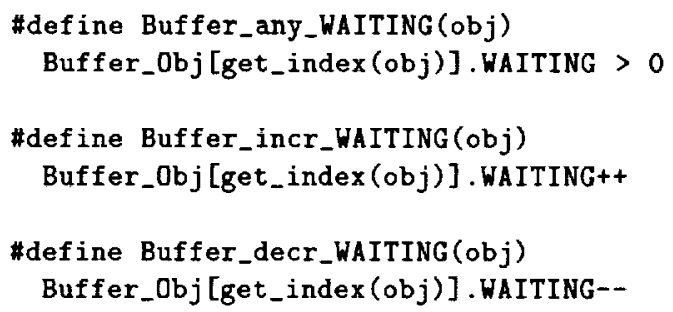

Fig. 13. Operations on WAITING

ables LOCK, WAITING and WAIT used for managing object locking and release. The LOCK variable will at any time either be nuIl (a negative integer) or it will be the thread id of the thread that currently is executing a synchronized method on the object (actually a non-negative Promela process id). Hence, once this field is set to a proper thread id by a thread that calls a synchronized method, only the thread with this thread id is allowed to operate on the object. When the call of the synchronized method terminates, the lock is released by setting it to null again. Note that a synchronized method must be allowed to call other synchronized methods on the same object without causing blocking.

The variables WAITING and WAIT are used to manage threads that call the wait, notify, and notifyAll methods on the object. A thread that calls wait() will first unlock the object, and then try to read a value on the zero place channel WAIT. A zero place channel in Promela is used to model rendezvous communication, hence some other thread must send a value on this channel in order for the calling thread to be released. At any time, all threads that are waiting to get access to the object are waiting on this channel. Each time a thread calls the wait () method on the object, the WAITING variable is additionally incremented, and conversely decremented when released. Hence, the value of this variable will always be the number of threads waiting on the object. The variable is used when notifyAll is called by a thread, and all waiting threads have to be released: we need to know how many times the WAIT channel must be signaled. The operations on the WAITING variable are shown in Figure 13.

Locking and unlocking an object is implemented by the operations shown in Figure 14. Locking an object 


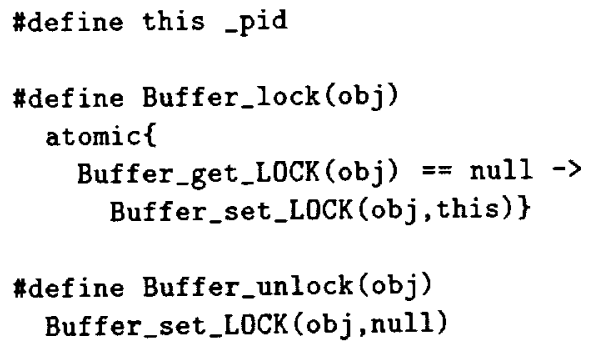

Fig. 14. Operations for locking and unlocking objects

is done when a thread calls a synchronized method on an object which the thread has not already locked. First the thread waits until the lock becomes free (no other thread has locked it), which is the case when the LOCK field becomes null. Note that in Promela, an equality is a statement that is executable only when the equality holds, and hence blocks until this is the case. Then, in an atomic move, the thread locks the object by assigning the value of "this" to the LOCK field, where "this" denotes the thread id of the current thread ("this" is defined as -pid, which in Promela refers to the process id of the currently executing process). Unlocking an object just corresponds to setting the LOCK field to null.

Finally, the macros modeling the wait, notify and notifyAll methods are shown in Figure 15. The Buffer-wait macro models the wait method. Ignore for a moment the unless constructs. When called, it first unlocks the object, because other threads should now have access to perhaps later call notify. Then the WAITING field is increased, and finally the thread starts waiting for a signal (the value 0 ) on the WAIT channel. When the thread is woken up by a notify or notifyAll, it locks the object again (first occurring call of Buffer_lock $(o b j)$ ) in order to continue with exclusive access to the object (note that wait can only be called within a synchronized context in JAVA).

The Buffer_notify macro signals the WAIT channel in case there are threads waiting for a signal. The Buffer_notifyall macro does this as long as there are threads waiting. Note that this happens atomically so that no new threads can join the waiting threads during the notification.

Now we explain the unless constructs in the Buffer_wait macro. This extra complication is caused by the fact that another thread can either interrupt or stop a thread that is waiting. Recall that such an interrupt or stop is like an exception being thrown inside the (perhaps waiting) thread being interrupted or stopped. We shall later explain exceptions in more detail, but here it suffices to say that in case such an exception is thrown, the waiting thread must be released immediately; this occurs because the conditions of the two unless constructs become true.

Suppose that the waiting thread is blocked in the "Buffer_get_WAIT (obj)? continue" statement, and that

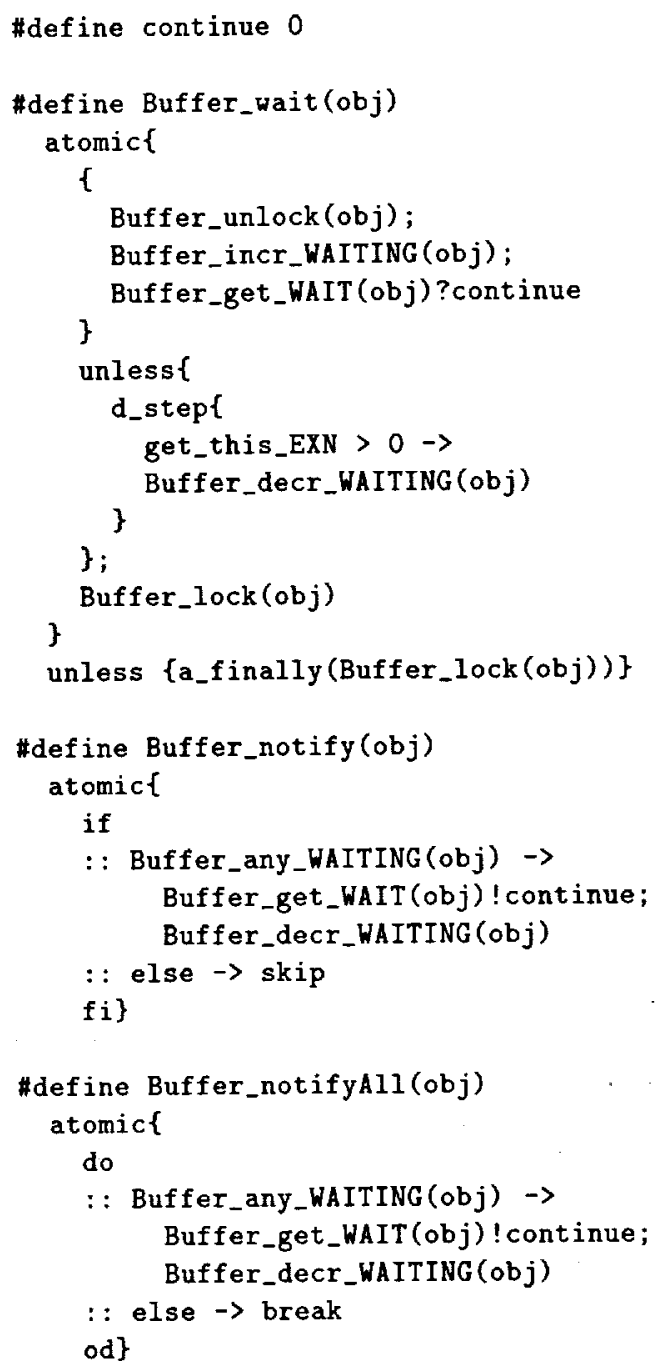

Fig. 15. The operations wait, notify and notifyAll

such an interrupt occurs. Then (due to the new semantics of the Promela unless construct, see Section 3.5) the inner unless construct will be triggered since, as we shall see, this implies that get_this_EXN becomes bigger than zero. This causes the WAITING variable to be decremented, which is necessary because the Buffer notify and Buffer_notifyAll macros normally do this (and now have not had the chance to do it). Next, because the waiting thread may have code in finally constructs that must be executed before termination (not shown here), it is necessary to lock the object again before stopping such that this finalize code can be executed with exclusive access to the object. This is done by the outermost unless construct. It was necessary to introduce two unless constructs since the interrupt may also arrive after the thread has been woken up, but while it is waiting for the lock to be released; that is, it is waiting in the first occurring Buffer_lock (obj) statement. 


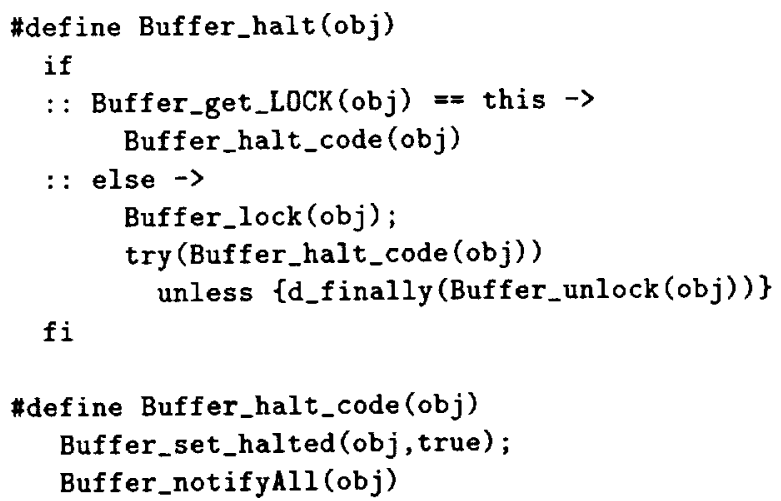

Fig. 16. Translation of the halt method

\subsection{Methods}

In order to illustrate how methods are modeled, we illustrate the simplest one, namely the halt method. A synchronized method $M$ in a class $C$ is defined in two parts: a C_M macro dealing with object locking, and a C_M_code macro containing the actual code of the method. For non-synchronized methods there is no such distinction. This is shown for the synchronized halt method in Figure 16. In general, a method call is translated into a call of the C.M macro with parameters corresponding to the method parameters. In the case of halt, the method is parameterless, hence only the object reference of the object upon which the method is called is given as parameter. The macro tests whether the object is already locked by the calling thread, in which case the actual code, C_M_code, is executed. This models the situation where this method is called by another synchronized method, hence the object is already locked. In case the object is not locked by the calling thread, an attempt is made to lock it (Buffer lock (obj)), and after successful locking, the code is executed. The try macro models the try construct of JAVA and models the fact that the object must always be unlocked (Buffer_unlock(obj)) when leaving the method, also in the case where an exception is raised during execution of the code.

\subsection{Threads}

It remains to explain how things are put together by translating the main method and the threads that it starts. First, we describe how the thread classes Producer and Consumer are translated. We focus on the Producer. Thread classes are translated in a similar manner to other classes, with the addition that the run method is translated into a Promela process type (proctype), that can then be started using the Promela run statement, whenever a corresponding JAVA thread is started with the JAVA start method. Figure 17 shows the translation of the run method of the Producer class of Figure 5.

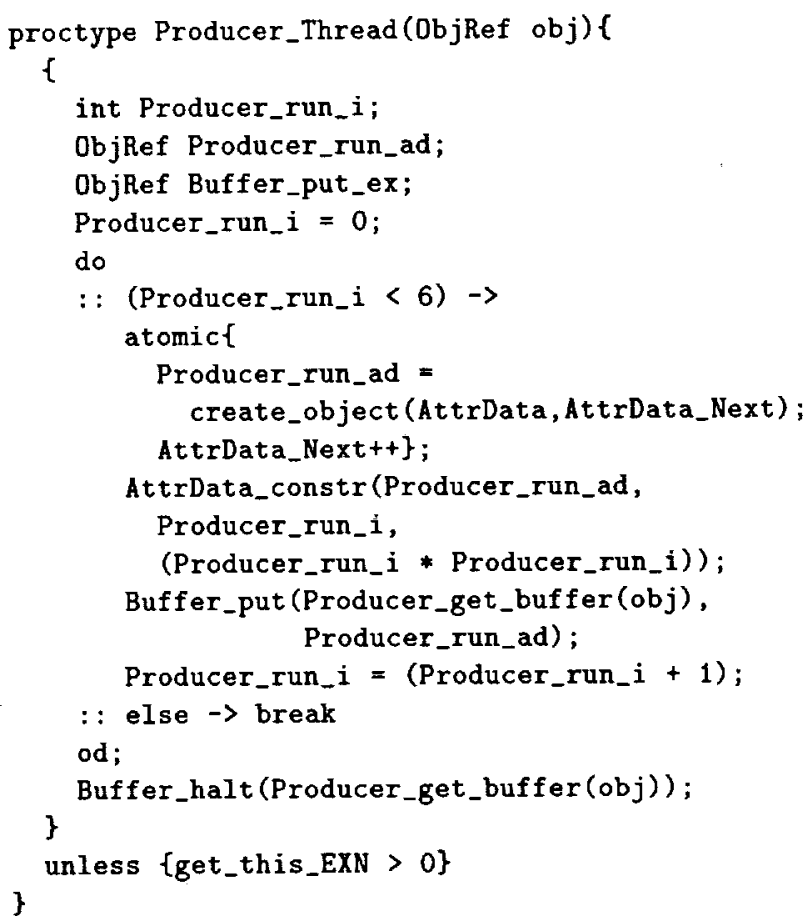

Fig. 17. The Producer run method

The process takes as parameter its own object reference. Notice how local variables in called methods are declared as global variables in the process. For example, the variable ex in the put method that binds the caught exception in the try statement (Figure 2) is declared at this point. This is necessary because Promela does not support local variables (nor does it in the new inline procedures that Promela got recently). This solution of course prevents the translation of recursive methods, but it is efficient with respect to verification time since we don't need to maintain a call stack (and represents what one normally would do in a hand translation). Another possibility is to translate methods into proctypes, as suggested in [15], but experiences during earlier work suggested that this would be inefficient, see [9]. The while loop is translated into PromelA's do ... od construct in a very straight forward way.

Note in Figure 17 how the object reference to the buffer in the buf fer variable declared inside the Producer class is accessed and passed as argument to the put and halt methods. This models the dot-notation in $\mathbf{J}_{\text {AVA }}$ for accessing methods in an object. Surrounding the producer thread code is an unless construct, which is supposed to catch any exceptions thrown and not caught by the user program. This will be explained in the next section. Finally, Figure 18 shows the translation of the Producer object constructor. It shows how the buffer variable is initialized with the argument, and how the process is started, corresponding to the "this.start()" call. 


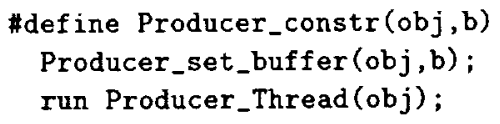

Fig. 18. The Producer constructor

\subsection{Exceptions}

JAVA exceptions are complicated when considering all the situations that may arise, such as method returns in the middle of try constructs, the finally construct, interrupts (which are exceptions thrown from one thread to another) of threads that have called the wait method, and the fact that objects have to be unlocked when an exception is thrown out of a synchronized method. We shall try to illustrate our solution.

Promela's unless construct seems very closely related to an exception construct, except for the fact that it works "outside in" instead of "inside out", the latter being the case for exceptions. As an example consider the JAVA statement (assuming some variable $\mathbf{x}$ ):

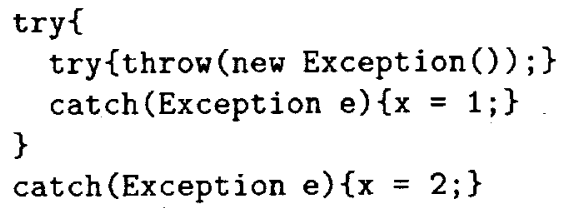

The result of executing this statement should be that $\mathrm{x}$ is assigned to 1 , hence (only) the inner catch is invoked when the exception is thrown. In contrast, consider a rclated Promela statement, where now an EXN variable (initially 0 ) has been introduced. When this variable becomes positive it is regarded as if an exception has been thrown:

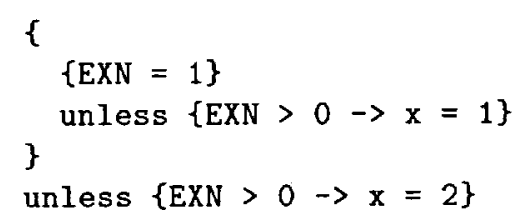

The effect of this statement will be that $\mathrm{x}$ is assigned to 2 . Hence, the outermost unless construct is invoked when EXN becomes positive. Gerard Holzmann has been very helpful to us by implementing a $-J$ option ( $\mathrm{J}$ for J AVA) in the verifier that changes the semantics of Promela in such a way that unless constructs are interpreted "inside out". This still leaves a second issue: that as soon as the inner unless is chosen (executing the inner EXN > 0), then the outer is invoked (since EXN $>0$ is still true) and $x$ gets the value 2 anyway. Hence, we have to prevent that kind of behavior. Note that the above Promela code needs some modifications to actually "work" because if it is the last statement in "P" within the statement "P unless $Q$ " that makes " $Q$ " executable, then "Q" will actually not get executed.

Recall that a JAVA exception is an object of one of the exception classes, either one of the built in or a user

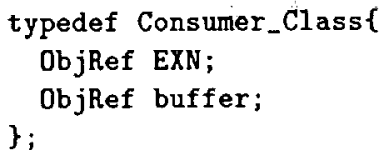

Fig. 19. The Consumer object type

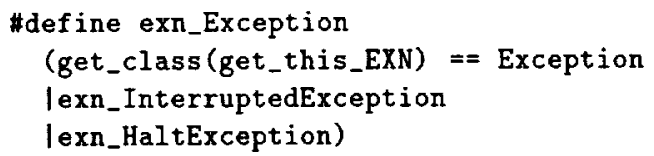

Fig. 20. The exn_Exception predicate

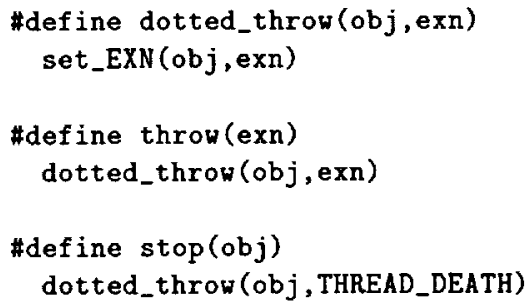

Fig. 21. Operations for throwing exceptions

defined exception class. Hence, an exception may contain data and methods. The throw(e) statement takes an exception object reference e as argument, and in our model we translate that into a store of that object reference in a special variable, EXN, in the data area of the thread (potentially the main program) where it has been thrown. All the unless constructs in the thread will test on this variable to see if it becomes different from nu1l. The data area for the Consumer class is shown in Figure 19. There are corresponding macros for accessing this variable. There are macros get EXN (obj) and set EXN (obj, value) for accessing this variable of a thread identified by obj. In addition, the macros get_this_EXN and set_this_EXN will access the EXN variable of this thread.

For each exception class $E$, there is a predicate exn $E$ which evaluates to true if the EXN variable contains an exception object of that class or a subclass thereof. For example, Figure 20 shows this predicate for the built in Exception class. Recall that the HaltException was defined as a subclass thereof, as is InterruptedException.

Exception throwing is mainly modeled by the macros shown in Figure 21. The dotted_throw macro throws an exception at a particular object given as parameter. This allows one thread to throw an exception at another thread, causing that other thread's EXN variable to be set. The throw macro throws an exception to the object identified by the free variable obj which is this current thread object. Finally, calling "T. stop()" in a JAVA program corresponds to throwing the predefined THREAD DEATH exception object to the T object.

We shall now explain how exceptions are caught. Consider a try statement of the form: 


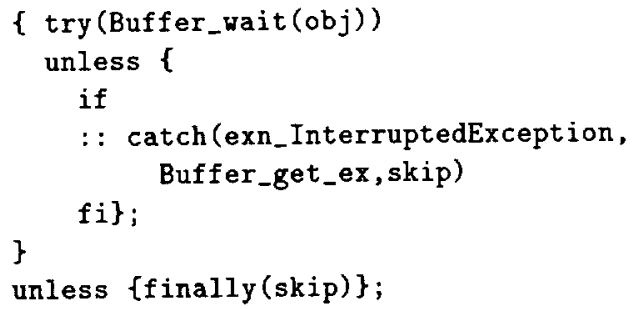

Fig. 22. Translation of the try statement in the get method

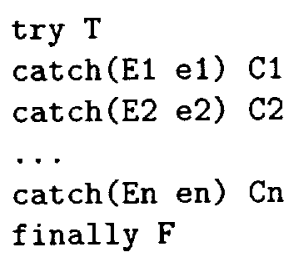

A JAVA statement of this form is translated into a Promela statement of the following form using macros defined in Figure 23, and where a prime (') after a block indicates its translated version:

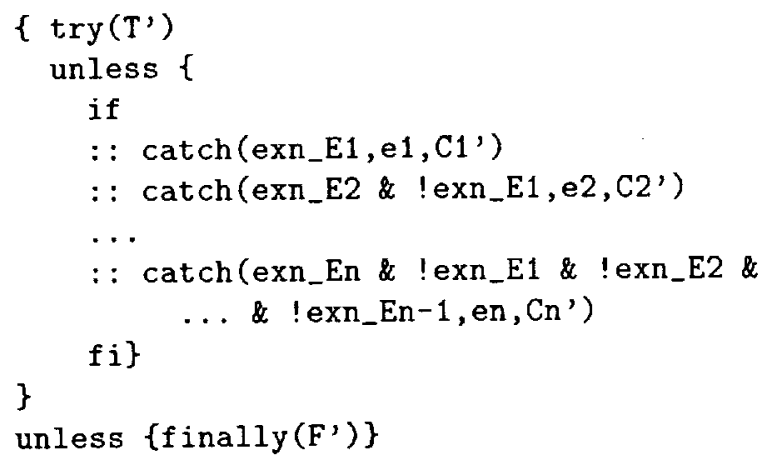

For example, the try construct in the get method in Figure 2 is translated into the Promela code in Figure 22. The general idea behind this translation is that the inner unless construct catches any exceptions which match any one of the exception predicates exnE1 ... exn En, and that the outer unless construct models the finally construct in JAVA: the block $F$ must be executed no matter what as the last thing. For example, in the case where for example $\mathrm{C} 1$ ' itself throws a new exception, the $F$ still has to be executed before that exception can be thrown further up. Now, let us explain the macros in Figure 23.

The try (s) macro executes $s$ and then throws the predefined EXIT object (exit_to_final). The EXIT exception is then caught by the finally macro (always inserted at the end) as we shall see. The catch (exn E, $x, s$ ) macro tests whether the current value of the EXN variable satisfies the exn $\mathbf{E}$ predicate (catch_cond (exn_E, $\mathrm{x}$ )), in which case that branch is executed.

The catch_cond (exn E,e) macro in an atomic move tests the predicate exn $E$ (hence is only executable when it is true, see Figure 20 for one such predicate), and then stores the value of the exception in the local variable e to

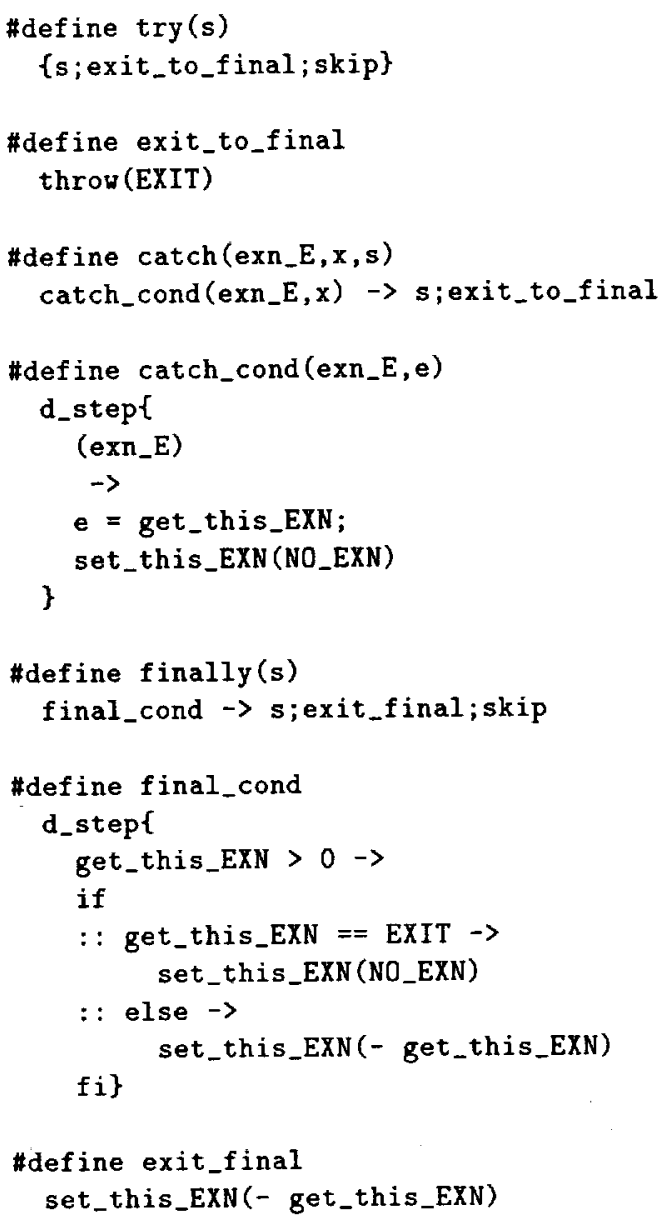

Fig. 23. Operations for catching exceptions

be accessible within the block to be executed. Note also how the EXN variable is zeroed to the predefined NOEXN value in order to avoid outer unless constructs being triggered, as already discussed earlier on page 10 .

The finally (s) macro defines when the outer unless construct should be triggered, namely when final_cond becomes executable. The $f$ inal_cond macro is executable whenever there is some exception object reference in the EXN variable (it is bigger than 0 ). Note that we reset this to 0 (NO_EXN) whenever an exception is caught at an inner level so that this outer final construct will not get activated. If there is an exception, and this is just a normal EXIT from one of the blocks T,C1, . , Cn in the try statement, this is then forgotten (set_this EXN (NOEXN)). If on the other hand (else) it concerns an exception that has not been caught, we need to remember it so that it can be re-thrown after the code in the finally construct has been executed. We also need to make sure that it does not activate unless constructs higher up during the execution of the finally code. One way to do this is to negate it since the unless constructs in the translation only react on positive values of EXN. When we then leave the finally construct, we negate it back to its positive value such that it can be "thrown" fur- 


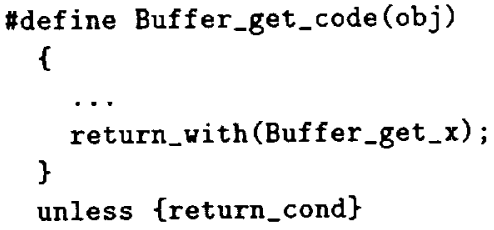

Fig. 24. Translation of the value returning get method

ther up. Some of the macros contain skip statements. These are necessary in order to make it work due to the semantics of the unless construct.

In Figures 15 and 16 the macros a_finally(s) and d_finally (s) are called. These are defined as respectively atomic $\{\mathrm{finally}(\mathrm{s})\}$ and d_step $\{\mathrm{finally}(\mathrm{s})\}$.

The d_finally (Buffer_unlock(obj)) call in Figure 16 unlocks the object in case an exception has been thrown out of a synchronized method. The d_step ensures that the exception is not thrown further up during the unlocking (which is more economic than negating the exception) and the unlocking can be made completely atomic since there are no blocking statements.

The a.finally(Buffer lock(obj)) call in Figure 15 locks an object after a call of wait has been interrupted. This locking must be as atomic as possible, but with the possibility of blocking since another thread may own the lock. Therefore a d_step cannot be used (it does not allow blocking).

\subsection{Value Returning Methods}

A JAVA return statement can have one of two forms. Either it has the form "return" in case the method is not value returning, or the form "return exp" if the method is value returning. In either case, such a return statement has the same effect as throwing an exception that is caught by a "return exception handler" surrounding the body of the value returning method, and by any finally constructs on the "way up" to that. Figure 24 shows how the get method of the Buffer class translates with respect to value return.

We see that the body of the method is surrounded by an unless construct triggered by the return_cond predicate. This predicate, defined in Figure 25, becomes executable when the EXN variable in the executing thread (the one that executes the value returning method) gets the value RETURN which is a predefined fixed object reference. This happens for example when the return_with macro is called with an argument denoting the value returned. The return_without models the return from a method that does not return a value.

The assignment "RES [TOP] $=\mathrm{e}$ " in the return_with macro needs some explanation. The problem we are faced with is that JAVA allows value denoting expressions to contain calls of value returning methods that may have side effects. In Promela expressions are of a syntactically different class than statements, and cannot have

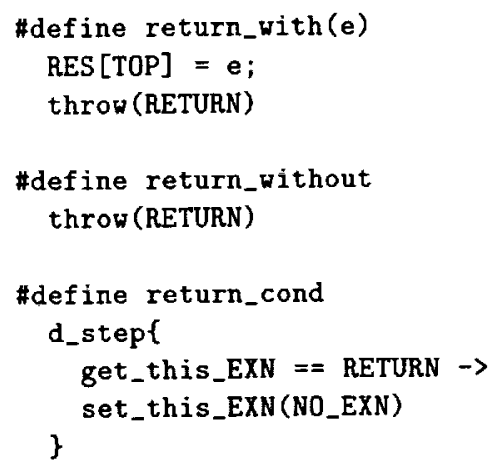

Fig. 25. Operations for return exceptions

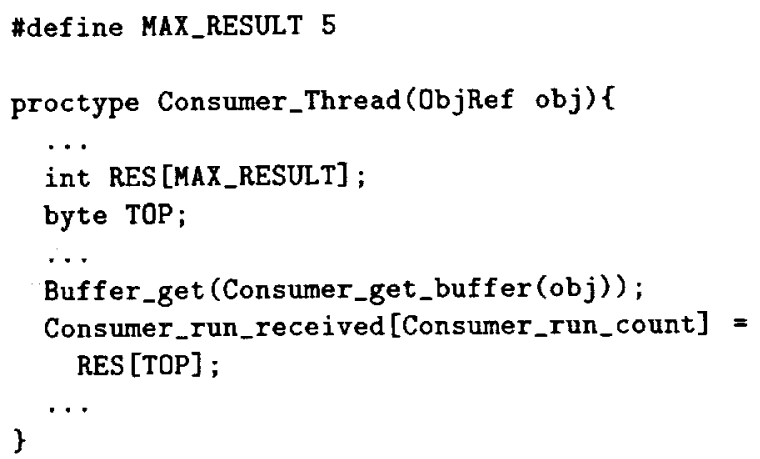

Fig. 26. The Consumer thread

side effects. Consider for example the following statcment in the Consumer class's run method:

$$
\text { received [count] }=(\text { AttrData }) \text { buffer } \cdot \operatorname{get}() \text {; }
$$

Ignoring the casting "(AttrData)...", the right hand side of this assignment has side effects, whereas the right hand side of a Promela assignment does not allow this. Our solution is to execute the method before the assignment, and then store the result value in a result array that is introduced for this purpose. Note that it has to be an array in order to model assignment statements of the form " $x=0 \cdot m(x)+p \cdot n(y)$ " where several value returning method calls occur in an expression. Part of the translation of the Consumer class's run method is shown in Figure 26. The Buffer get macro will execute and finally store the return value in RES[TOP], where RES is the result array. This value is then "picked up" in the assignment statement. Note that if there are several value returning method calls in a single expression, the TOP variable is incremented for each during their evaluation, and then reset to its previous value before the values are accessed.

\subsection{The main Method}

The main method of the Main class is translated into the Promela init section as can be seen in Figure 27 . We have already explained the translation of object creation, which is the only activity of this particular main 


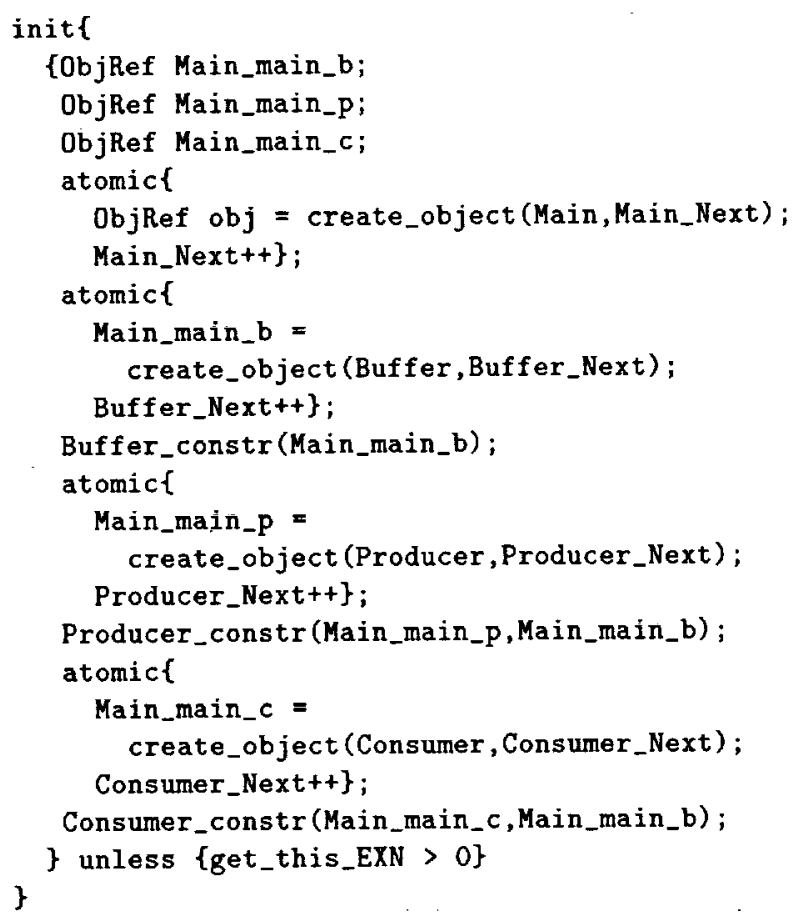

Fig. 27. The main method

method. Recall that the constructors of the Producer and Consumer classes in this present example start the processes.

\section{Analyzing the Program}

The presented program is correct in the sense that no errors are found by SPIN when applied to the Promela code generated by JPF. In order to illustrate the effectiveness of JPF (and SPIN of course) we have seeded 21 errors in the program shown in Figure 2, and for each error analyzed the now incorrect program using JPF. This experiment is described in the following.

\subsection{The Result}

The results of this experiment are shown in Table 1 . For each error we give the line numbers changed, referring to Figure 2, and the new contents of these lines. As an example, error 1 is obtained by changing line 3 to "protected int putPtr $=1$; " (initializing to 1 instead of to 0 ).

The results of applying JPF are shown in the fourth column. That is, the result of applying the SPIN model checker to the Promela code generated by JPF. The possible outcomes are deadlock (D) and any of the two assertions being violated (A1 referring to the first occurring "count $==6$ " and A2 referring to the second "received[i] .attr == i"). The point here is that all the errors are caught.
The last two columns show the result of running the modified JAVA program on two versions of the JAVA Virtual Machine (JVM) in order to see whether plainly executing the program would highlight the errors seeded. JVM version 1.1.3 is an older version being very deterministic. This means that executing a multi-threaded program several times typically yields the same result every time. JVM 1.1.6 is the newer version with native threads, where JAVA threads are mapped to Solaris threads. This version is therefore non-deterministic, potentially yielding different results for different runs of a multi-threaded program.

Every program has been run several times (from 30 to 100), and the numbers indicate the percentage of runs that have highlighted the error, either via a deadlock, an assertion violation, or a thrown NullPointerException.

All runs, model checking as well as JVM runs, have been executed on a Sun Ultra Sparc 60 with $512 \mathrm{Mb}$ of main memory, and with the Solaris operating system version 5.5.1.

Running the SPIN model checker on the Promela code generated by JPF typically used less than half a second to find an error and explored between 40 and 400 states and a similar number of transitions. In a few cases (error 8 and 10) approximately 10000 states and 18000 transitions were explored in less than 2 seconds. The memory consumption was around $17 \mathrm{Mb}$. This amount of memory must probably be explained by the modeling of the Java Virtual Machine within Promela.

"Errors" 11 and 20 are special (marked with a $*$ ) in the sense that they are not really errors when using the environment described in Section 2.2. This environment only creates one consumer, and to make the errors manifest themselves, we needed to create two consumers. In addition, with two consumers the assertions make no sense and were deleted. Hence, we were now just looking for deadlocks. The table rows for these errors show the result of verifying and executing in this changed multi-consumer environment. The verification of error 11 needed as much as 8 minutes and $77 \mathrm{Mb}$, exploring 2.4 million states and 6 million transitions before the deadlock was found. We verified a down scaled version of this error, with a buffer size of 2 (instead of 3 ) and the producer only producing 3 values (instead of 6 ). Also here the deadlock was found by the model checker, but now using 1 minute, $28 \mathrm{Mb}$, and exploring 423096 states and 1 million transitions.

SPIN used 5.2 seconds to verify that the original program contained no errors. This involved the exploration of 33683 states and 61944 transitions, and a memory consumption of $18.8 \mathrm{Mb}$.

\subsection{Comments on the Result}

The example is small since its main purpose has been to illustrate the translation done by JPF. However, the exercise does show that around half (11) of the errors 
Table 1. Verification results

\begin{tabular}{|c|c|c|c|c|c|}
\hline Nr. & Line & Modification (changed to) & JPF & JVM 1.1 .6 & JVM 1.1 .3 \\
\hline 1 & 3 & protected int putPtr $=1$ & A2 & 100 & 100 \\
\hline 2 & 5 & protected int usedslots $=1$; & $\mathrm{A} 1, \mathrm{~A} 2$ & 100 & 100 \\
\hline 3 & 9 & while (usedSlots $!=3$ ) & $\mathrm{D}$ & 100 & 100 \\
\hline 4 & 9 & while (usedSlots $==2$ ) & $\mathrm{D}$ & 65 & 0 \\
\hline 5 & $\begin{array}{l}12 \\
13\end{array}$ & $\begin{array}{l}\text { putPtr }=(\text { putPtr }+1) \% 3 ; \\
\text { array }[\text { putPrt] }=x\end{array}$ & A2 & 100 & 100 \\
\hline 6 & 13 & putPtr $=$ putPtr $\% 3$ & $\mathrm{~A} 2$ & 100 & 100 \\
\hline 7 & $\begin{array}{l}13 \\
28\end{array}$ & $\begin{array}{l}\text { putPtr }=(\text { putPtr }+1) \% 2 \\
\text { getPtr }=(\text { getPtr }+1) \% 2\end{array}$ & $\mathrm{~A} 2$ & 56 & 0 \\
\hline 8 & 14 & if (usedSlots $==3$ ) notifyAll(); & $\mathrm{D}$ & 33 & 100 \\
\hline 9 & 14 & $\begin{array}{l}\text { remove: } \\
\text { if (usedSlots }==0 \text { ) notifyAll(); }\end{array}$ & $\mathrm{D}$ & 55 & 100 \\
\hline 10 & $\begin{array}{l}14 \\
15\end{array}$ & $\begin{array}{l}\text { usedSlotst+; } \\
\text { if (usedSlots }=0 \text { ) notifyAllo); }\end{array}$ & $\mathrm{D}$ & 35 & 100 \\
\hline $11^{*}$ & $\begin{array}{l}14 \\
29\end{array}$ & $\begin{array}{l}\text { if (usedSlots }==0 \text { ) notify (); } \\
\text { if (usedSlots }==3 \text { ) notify (); }\end{array}$ & $\mathrm{D}$ & 2 & 100 \\
\hline 12 & 20 & while (usedSlots $==0$ ) & $\mathrm{D}$ & 100 & 100 \\
\hline 13 & 23 & if (usedSlots $!=0)\{$ & $\mathrm{A} 1, \mathrm{D}$ & 100 & 100 \\
\hline 14 & 23 & if (halted) \{ & A1 & 3 & 0 \\
\hline 15 & 29 & if (usedSlots $==0$ ) notifyAll(); & $\mathrm{D}$ & 50 & 0 \\
\hline 16 & 29 & $\begin{array}{l}\text { remove: } \\
\text { (if usedSlots }==3 \text { ) notifyAll(); }\end{array}$ & D & 44 & 0 \\
\hline 17 & $\begin{array}{l}29 \\
30\end{array}$ & $\begin{array}{l}\text { usedSlots--; } \\
\text { if (usedSlots }==3 \text { ) notifyAllo); }\end{array}$ & D & 66 & 0 \\
\hline 18 & 30 & usedSlots++ & $\mathrm{A} 1, \mathrm{~A} 2$ & 100 & 100 \\
\hline 19 & 35 & $\begin{array}{l}\text { remove: } \\
\text { halted = true; }\end{array}$ & $\mathrm{D}$ & 100 & 100 \\
\hline $20^{*}$ & 36 & notify () & $\mathrm{D}$ & 2 & 100 \\
\hline 21 & 36 & $\begin{array}{l}\text { remove: } \\
\text { notifyAll(); }\end{array}$ & $\mathrm{D}$ & 100 & 100 \\
\hline
\end{tabular}




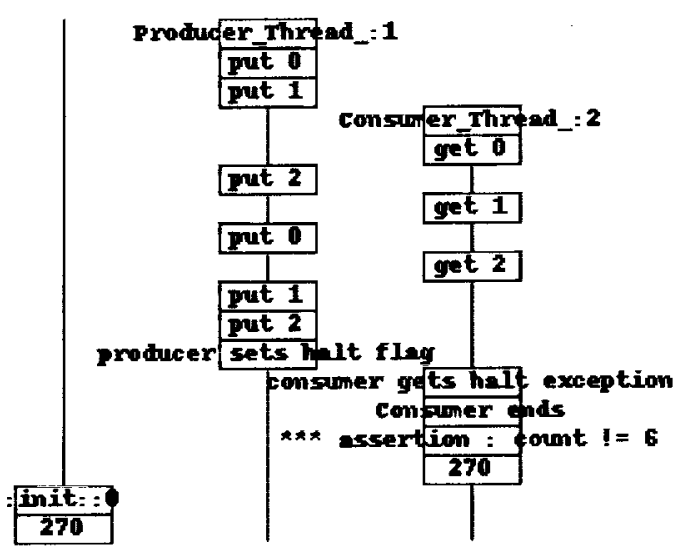

Fig. 28. Trace for error 14

are not guaranteed to be caught when plainly executing the program on the newest version of JVM, while in contrast JPF finds the errors each time if present in the setup (errors 11 and 20 were not present with only one consumer). The difference is most obvious for errors 11 , 14 and 20 . Errors 3 and 11 were suggested by a reviewer. The chance of catching errors by executing the program gets even smaller when the program size increases.

\section{$4.3^{2}$ Error Traces}

When SPIN identifies an error in the translated Promela code, be it a deadlock or an assertion violation, it returns with an error trace showing the sequence of executed Promela statements leading from the initial state to the state where the error occurs. Our tool does not currently map such Promela error traces back to corresponding JAVA traces. As an alternative, we have extended the Verify class with special print methods which can be called (by the programmer) in selected places in the JAva code. These calls will then be translated into Promela print statements set up to print on a graphical two dimensional message sequence chart. Figure 28 shows such a chart illustrating the trace for error number 14 .

To obtain this chart, to be explained below, we have added print statements 5 places in the code. For example, in lines 12 and 26 (pushing current lines downwards) we have added the statements:

$$
\begin{aligned}
& 12 \text { Verify.print("put",putPtr); } \\
& 26 \text { Verify.print("get",getPtr); }
\end{aligned}
$$

Hence, what will be printed out is a text string ("put" or "get"), and the position in the buffer where a value is stored (put), respectively retrieved from (get). Other print statements record other events, such as the producer setting the halted flag, and the consumer getting the corresponding exception. Figure 28 illustrates a situation where the producer (center vertical line) first produces two values, stored in positions 0 and 1 . Then the consumer gets the value in position 0 , etc. The consumer only gets the first three values. The producer then finishes and sets the halted flag, where after the consumer now gets an exception when trying to get the fourth value (in position 0 ). Therefore the consumer will miss three values, and the first assertion (count $==6$ ) will be broken. Note that the second assertion is not broken.

Adding these print statements requires thought and work, but the idea may in fact be useful to cut down the enormous amount of information contained in the error traces created by SPIN.

\section{Conclusion and Future Work}

In this paper we have described the initial prototype of a translator from a non-trivial subset of the general purpose programming language $J_{A V A}$ to the model checking language Promela. We have also applied the translator to 21 bugged variations of a small example program, observing that the model checker catches all the bugs, while executing the programs only catch the bugs safely in half of the cases.

We are currently applying the translator to a collection of real programs developed within NASA, and the outcome of these experiments will be published at a later time. As an example, a collaboration with NASA's Goddard Space Center involves the verification of a satellite file down-link protocol written in JAVA. The translator has in addition been applied to analyze a Chinese Chess game server application written in JAVA. This lead to the confirmation of a suspected deadlock and the identification of a smaller scenario leading to that deadlock. This work is documented in [13].

Although the translator covers a non-trivial subset of JAVA some important features are not covered. These are: packages, overloading, method overriding, recursion, strings, floating point numbers, static variables and methods, some thread operations like suspend and resume, some control constructs, such as the continue statement, and garbage collection. In addition, arrays are not objects as they are in JAVA. We model arrays using PROMELA's own arrays to obtain efficient verification. The current implementation thus does not allow arrays to be first class values and they cannot be multidimensional. Finally, we do not translate the predefined class library. Some of these features are hard to translate, such as recursion, strings, floating point numbers, and garbage collection. The rest are relatively straight forward, although an efficient translation of dynamic arrays as objects is not evident. We continue to extend the translator to cover more of J AVA.

An essential question is whether the translation is optimal. We still need to evaluate this question. There may be other ways to translate into Promela and one may consider making a by-pass, avoiding Promela, and translating directly into the $\mathrm{C}$ interface to the SPIN model 
checker. A major point of discussion has been how to model object creation. One obvious solution would be to use Promela's proctypes to model classes, and then spawn a process for each object creation. This would indeed help at the level of memory allocation and result in a simpler translation than into arrays. However, it conflicts with the fact that several threads can access the same object concurrently: a Promela process only has one thread of control.

Recently JPF has been modified to translate object synchronizations and exceptions more efficiently by introducing two new special purpose arrays holding locking and exception variables, thus keeping these variables separate from user defined variables. This gave a $50 \%$ reduction in the amount of $\mathrm{C}$-code generated by SPIN. We have presented the simpler solution for readability reasons.

We see the current translator as a phase 1 prototype experiment (involving 9 man months until now) that provides us with useful input to a phase 2, where these questions will be raised. What is equally important is our planned efforts to build an abstraction workbench around JAVA, where programs can be reduced in size before model checking is applied. Techniques such as program slicing, abstract interpretation, and partial evaluation will have a great influence.

We believe that the kind of technology presented in this paper, already as is, can be very useful for unit testing where one focuses on a single or a few classes, just as has demonstrated with the example. This requires setting up an aggressive environment consisting of a collection of threads which will "bombard" the unit with accesses. The technology is probably ready for this already now, since there will typically not be a big need to cut down the state space. Finally, we believe that perhaps the technology can be useful for students learning to program in JAVA.

Concerning the specification language, our main approach has been not to extend the JAVA language but to express temporal properties as calls to methods defined in a special temporal logic class (the Verify class), all of whose methods are static (hence one does not need to instantiate the class to objects before calling the methods). In addition to the assert method one can for example imagine an always method, an eventually method, and basically include all of SPIN's linear temporal logic operators as methods, having boolean return types in addition to boolean argument types, such that they can be composed. Calls of such methods will then generate LTL formulae to be verified, referring to the position where they are called in the code.

Acknowledgements. We thank Gerard Holzmann for changing SPIN to support the translation of exceptions. We thank the members of the Automated Software Engineering team at NASA Ames Research Center for their comments; and in particular Mike Lowry for supporting the project, and John
Penix and Willem Visser for proof reading the manuscript. We also thank the reviewers. We have used a JAVA parser ported by Peter Sestoft (the Royal Veterinary and Agricultural University in Denmark) to Moscow ML from a Standard ML version written by Olivier Brunet and Gordon Woodhull in Alex Aiken's group (University of California, Berkeley, USA).

\section{References}

1. K. Arnold and J. Gosling. The Java Programming Language. Addison Wesley, 1996.

2. R. Iosif C. Demartini and R. Sisto. Modeling and Validation of Java Multithreading Applications using SPIN. In Proceedings of the 4 th SPIN workshop, Paris, France, November 1998.

3. T. Cattel. Modeling and Verification of $\mathrm{sC}++$ Applications. In Proceedings of the Tools and Algorithms for the Construction and Analysis of Systems, Lisbon, Portugal, LNCS 1384., April 1998.

4. J. C. Corbett. Constructing Compact Models of Concurrent Java Programs. In Proceedings of the ACM Sigsoft Symposium on Software Testing and Analysis, Clearwater Beach, Florida., March 1998.

5. D. L. Delefs, K. R. M. Leino, G. Nelson, and J. B. Saxe. Extended Static Checking. Technical Report 159, Compaq Systems Research Center, Palo Alto, California, USA, 1998.

6. J. Gosling, B. Joy, and G. Steele. The Java Language Specification. Addison Wesley, 1996.

7. K. Havelund. Mechanical Verification of a Garbage Collector. In Fourth International Workshop on Formal Methods for Parallel Programming: Theory and Applications (FMPPTA'99), Lecture Notes in Computer Science, April 1999. To appear.

8. K. Havelund, K. G. Larsen, and A. Skou. Formal Verification of an Audio/Video Power Controller using the Real-Time Model Checker UPPAAL. In 5th Int. AMAST Workshop on Real-Time and Probabilistic Systems, Lecture Notes in Computer Science, May 1999. To appear.

9. K. Havelund, M. Lowry, and J. Penix. Formal Analysis of a Space Craft Controller using SPIN. In Proceedings of the 4th SPIN workshop, Paris, France, November 1998.

10. K. Havelund and T. Pressburger. Translating Java to SPIN, a step towards the JavaProver. Technical report, NASA Ames Research center, Moffett Field, California, USA, May 1997.

11. K. Havelund and N. Shankar. Experiments in Theorem Proving and Model Checking for Protocol Verification. In M-C. Gaudel and J. Woodcock, editors, FME'96: Industrial Benefit and Advances in Formal Methods, volume 1051 of Lecture Notes in Computer Science, pages 662-681, 1996.

12. K. Havelund and N. Shankar. A Mechanized Refinement Proof for a Garbage Collector. Submitted to the journal: Formal Aspects of Computing, 1998.

13. K. Havelund and J. Skakkebæk. Practical Application of Model Checking in Software Verification. Describes an application of JPF to a game server. Submitted for publication., February 1999. 
14. K. Havelund, A. Skou, K. G. Larsen, and K. Lund. Formal Modeling and Analysis of an Audio/Video Protocol: An Industrial Case Study Using UPPAAL. In Proc. of the 18th IEEE Real-Time Systems Symposium, pages 213, Dec 1997. San Francisco, California, USA.

15. G. Holzmann. The Design and Validation of Computer Protocols. Prentice Hall, 1991.

16. D. Lea. Concurrent Programming in Java. Addison Wesley, 1997.

17. B. Pell, E. Gat, R. Keesing, N. Muscettola, and B. Smith. Plan Execution for Autonomous Spacecrafts. In Proceedings of the 1997 International Joint Conference on Artificial Intelligence, 1997. 